\title{
THREE-DIMENSIONAL NUMERICAL SIMULATIONS OF THE COMBUSTION CHAMBER OF THE ROTATING DETONATION ENGINE
}

\author{
Michal Folusiak, Karol Swiderski \\ Arkadiusz Kobiera, Borys Lukasik, Piotr Wolanski \\ Institute of Aviation, Propulsion Department \\ Krakowska Av. 110/114, 00-256 Warsaw, Poland \\ e-mail:michal.folusiak@gmail.com
}

\begin{abstract}
From 2010 Warsaw University of Technology (WUT) and Institute of Aviation (IoA) jointly implement the project under the Innovative Economy Operational Programme entitled 'Turbine engine with detonation combustion chamber'. The goal of the project is to replace the combustion chamber of turboshaft engine GTD-350 with an annular detonation chamber. During the project, the numerical group that aims to develop computer code allowing researchers to simulate investigated processes has been established. Simulations provide wide range of parameters that are hardly available from experimental results and enable better understanding of investigated processes. Simulations may be also considered as a cheap alternative for experiments, especially when testing geometrical optimizations.

In this paper the analysis of simulation results of the combustion chamber of the Rotating Detonation Engine $(R D E)$ investigated at the IoA in Warsaw is presented. Primarily, REFLOPS USG which has become a fundamental numerical tool in the research of the RDE at the IoA is briefly described and governing equations and numerical methods used are shortly presented. Some aspects of numerical simulations of the RDE, related to selection of combustion mechanism, and an initiation of rotating detonation are provided. Secondly, results of simulations of inviscid gas with numerical injectors of hydrogen are compared with available experimental results. Three different wave patterns are identified in numerical solution and briefly described. Results of simulations are compared to experimental results in combustion chamber.

Results presented in this paper are part of the project UDA-POIG.01.03.01-14-071 'Turbine engine with detonation combustion chamber' supported by EU and Ministry of Regional Development, Poland.
\end{abstract}

Keywords: Rotating Detonation Engine, RDE, Computational Fluid Dynamics, CFD, REFLOPS

\section{Introduction}

In the following paper the analysis of results of three-dimensional simulations of the candidate combustion chamber of the Rotating Detonation Engine [6,8] investigated at Institute of Aviation in Warsaw is presented. Results of simulations of inviscid gas with numerical injectors of hydrogen are compared with available experimental results.

Simulations are based on the classical Euler equations, extended to describe the motion of a multicomponent reacting semi-ideal gas. The geometry is discretized on unstructured grid, and the conservation equations take the following form:

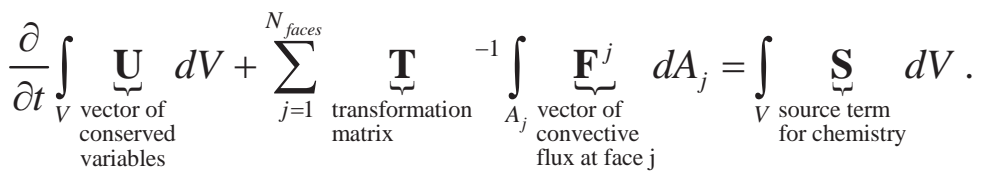

Numerical fluxes are evaluated by HLLC approximate Riemann solver [7]. Convective terms are integrated in time by use of the explicit Euler method. The source term $\mathbf{S}$ denotes chemical composition change rate resulting from the chemical reactions. The production rate of chemical compounds constitutes an overall sum of the production and destruction rates for a given chemical compound in all reactions taken into account in the chemistry model. The chemical reaction 
sources are integrated by the stiff ODE solver CHEMEQ2 [4] or DVODE [1]. The model is implemented into the in-house code REFLOPS USG [2, 3].

In the particular case presented in this work, combustion of hydrogen is simply described by one reversible reaction. The forward reaction rate is calculated by use of classical Arrhenius equation with the reaction rate constants verified against the Petersen 21-step mechanism [5] for the case of one-dimensional detonation wave propagation.

\section{Case description}

The considered combustion chamber of the RDE has single annular section of height 50 millimetres (see Fig. 1a). The injection of gaseous hydrogen is realized with 90 orifices of $0.7 \mathrm{~mm}$ diameter, placed perpendicularly to the main flow direction at the inner surface of throat. In the considered case pressure sensor is placed at the outer surface. The injection of Jet-A is realized with 24 injectors of $1 \mathrm{~mm}$ diameter, manufactured in convertible ring. However, in case of detonation simulations in pure hydrogen presented here, the ring is removed. The computational model of the high chamber was build up from 550000 hexahedral elements (see Fig. 1b), the smallest element size is $0.5 \mathrm{~mm}$. Numerical sensors and injectors are placed at positions corresponding to positions of real devices.
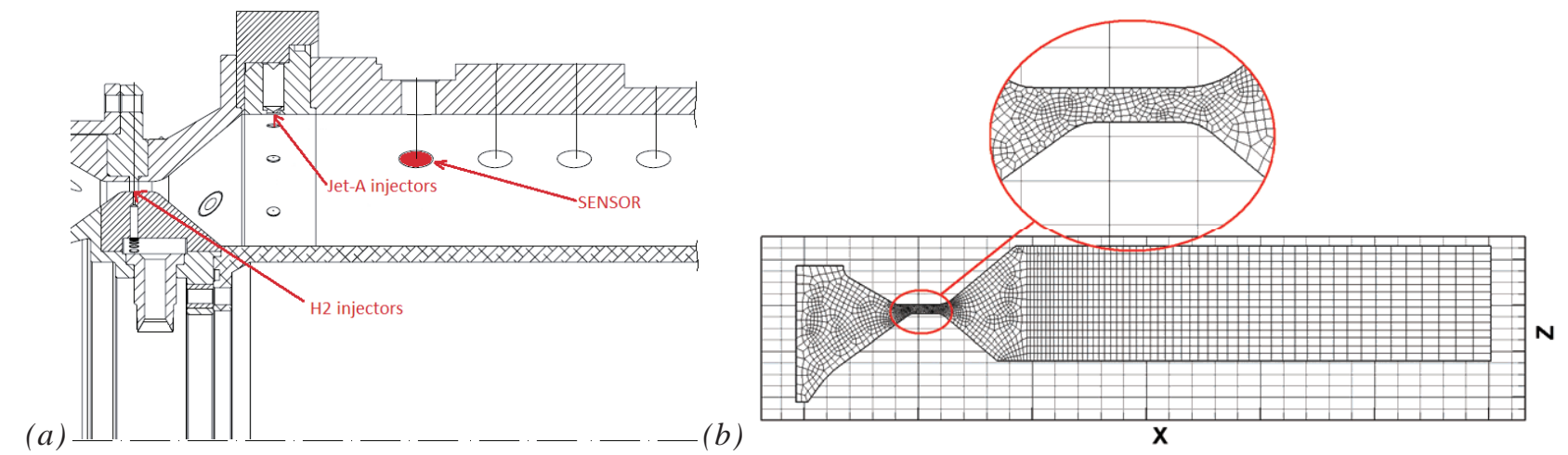

Fig. 1. (a) Geometry of the high combustion chamber of the RDE. The main flow direction is from left to right. (b) Longitudinal section of computational model of the chamber geometry

\section{Detonation analysis}

Simulations of hydrogen-air detonation have been performed for input conditions adopted from experiment, namely:

- inlet air pressure: 2.02 bar,

- inlet air temperature: $295 \mathrm{~K}$,

- mass flow rate of hydrogen: $13.42 \mathrm{~g} / \mathrm{s}$,

- resulting mass flow rate of air: $0.9285 \mathrm{~kg} / \mathrm{s}$.

Standard outlet conditions were assumed $\left(T_{\text {out }}=293.15 \mathrm{~K}, p_{\text {out }}=1.01325\right.$ bar $)$.

The most representative experimental results were selected and compared with results of simulation. Comparison of pressure sensor readings is shown in Fig. 2. It can be easily noted that pressure peaks measured by numerical sensors are higher than in experiment, which are mostly in the range of 1.5-1.8 bar. Both the height and the time separation of pressure peaks obtained in simulation are irregular, therefore it is investigated in detail below.

Assuming there are two detonation waves propagating in the chamber, the linear wave speed measured along the mean circumference $(0.635 \mathrm{~m})$ is about $1140 \mathrm{~m} / \mathrm{s}$ in numerical simulation and $1180 \mathrm{~m} / \mathrm{s}$ in experiment, while C-J velocity is $1511 \mathrm{~m} / \mathrm{s}$ in this case (see Tab. 1). The overall time of experiment was too short for thermocouple to reach the equilibrium temperature. However, it was 
found that the approximation of the transmittance of thermocouple with a second-order inertial term gives good results. The resulting outlet temperature is $T_{\text {outlet }}=518^{\circ} \mathrm{C}=791 \mathrm{~K}$ (see Tab. 1 ).

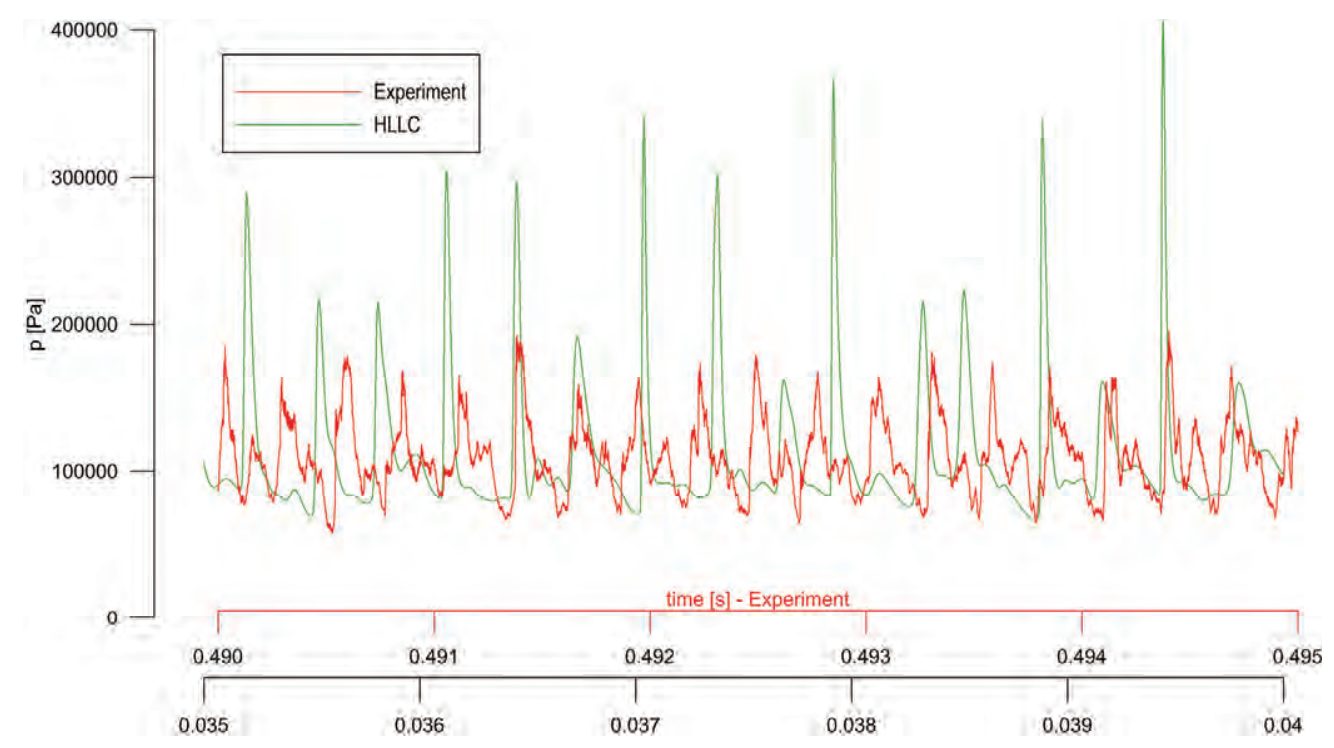

Fig. 2. Comparison of pressure profiles at sensors: experimental vs. numerical results

Tab. 1. Comparison of detonation parameters for experiment and simulation

\begin{tabular}{|c|c|c|c|}
\hline Case & $\begin{array}{c}\text { Temperature at outlet } \\
{[\mathrm{K}]}\end{array}$ & $\begin{array}{c}\text { Wave speed } \\
{[\mathrm{m} / \mathrm{s}]}\end{array}$ & $\begin{array}{c}\text { Mean pressure peak } \\
\text { ppeak }[\text { bar }]\end{array}$ \\
\hline Experiment & 791 & 1180 & 1.8 \\
\hline Simulation & 1050 & 1140 & 3.0 \\
\hline
\end{tabular}

Two additional numerical pressure sensors were placed in computational domain in order to explicitly identify number and direction of detonation waves (or strong shock waves) in the domain. The pattern of sensors is shown in Fig. 3.
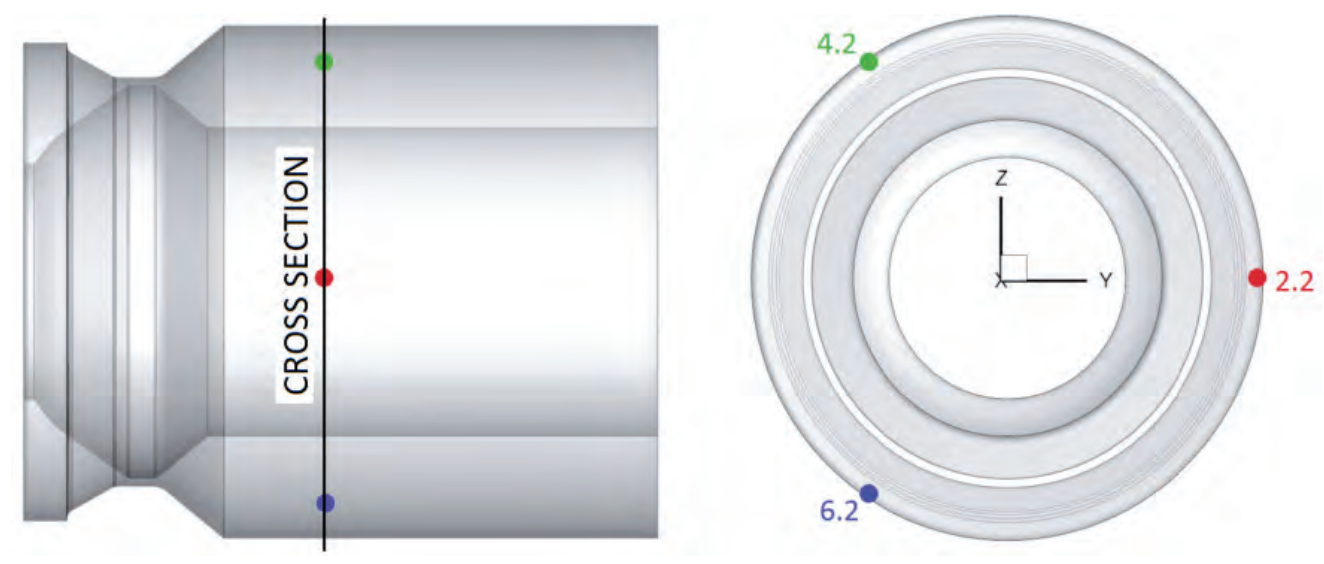

Fig. 3. Sensor location in the high combustion chamber of RDE

Three different wave patterns (denoted A, B and C) were identified in numerical solution and are shown in Fig. 4. Contour plots of static pressure at the plane defined by the three sensors are presented in Fig. 6. From these two figures, one can draw the following conclusions:

- in case A there are two detonation waves of similar strengths and speeds traveling in opposite directions (compare A1 and A2) that collide nearby sensor 4.2 (A3). Finally one of waves considerably strengthens (see A4 and A5), 
- in case B the stronger detonation wave from case A propagates through domain clockwise (see also Fig. 5) and strong shock wave propagates counterclockwise,

- in case $\mathrm{C}$, the direction of propagation of the two waves is opposite to B.

The state in the domain turns from one mode to the other continuously and no quasi-steady state solution is reached even for considerably long solution time. This behaviour may explain the observed irregularities in frequency of pressure peaks in experimental results.

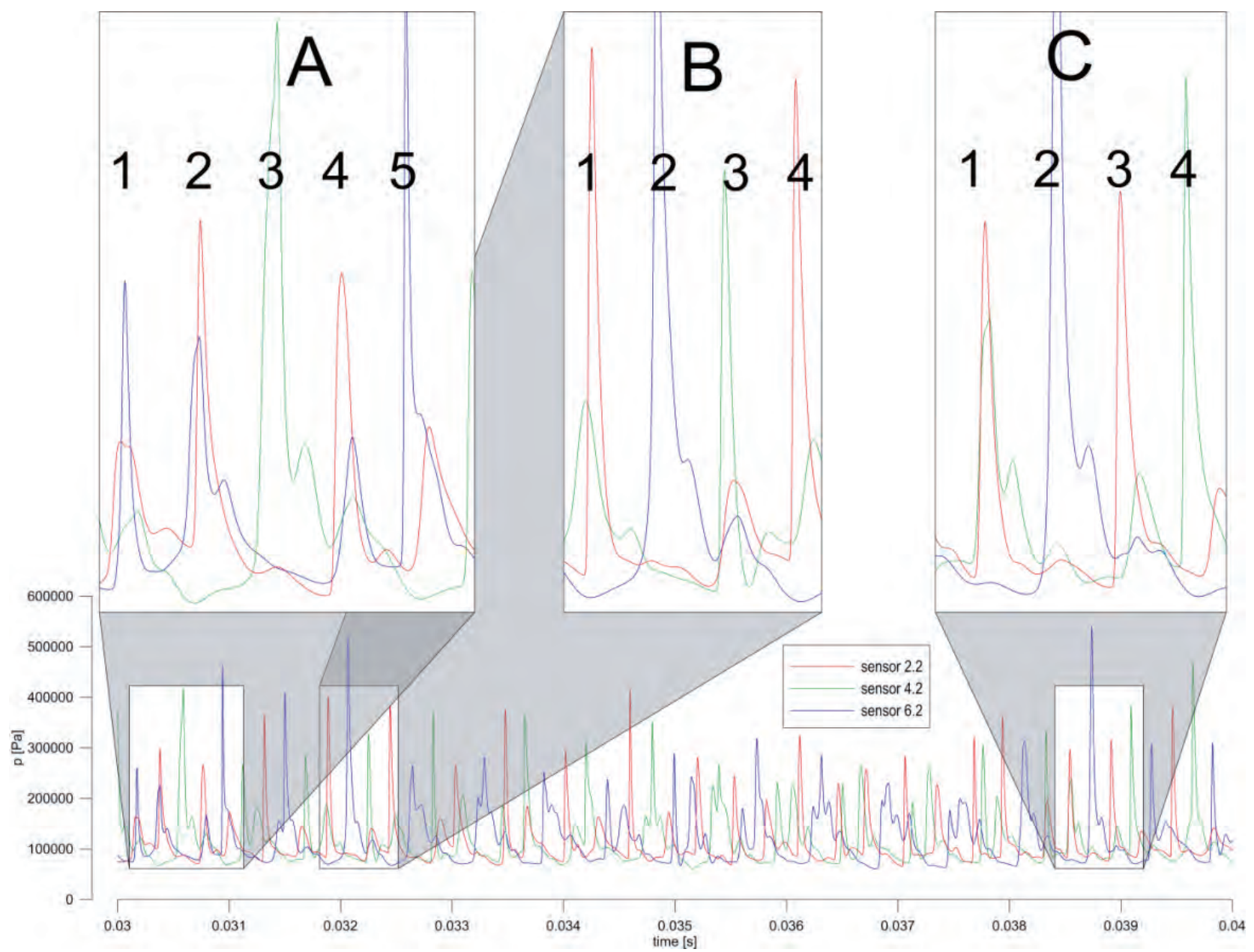

Fig. 4. Pressure sensor readings from REFLOPS USG for detonation case $p_{\text {in }}=2$ bar, $\lambda=2$ in high combustion chamber. Sensors located as shown in Fig. 3
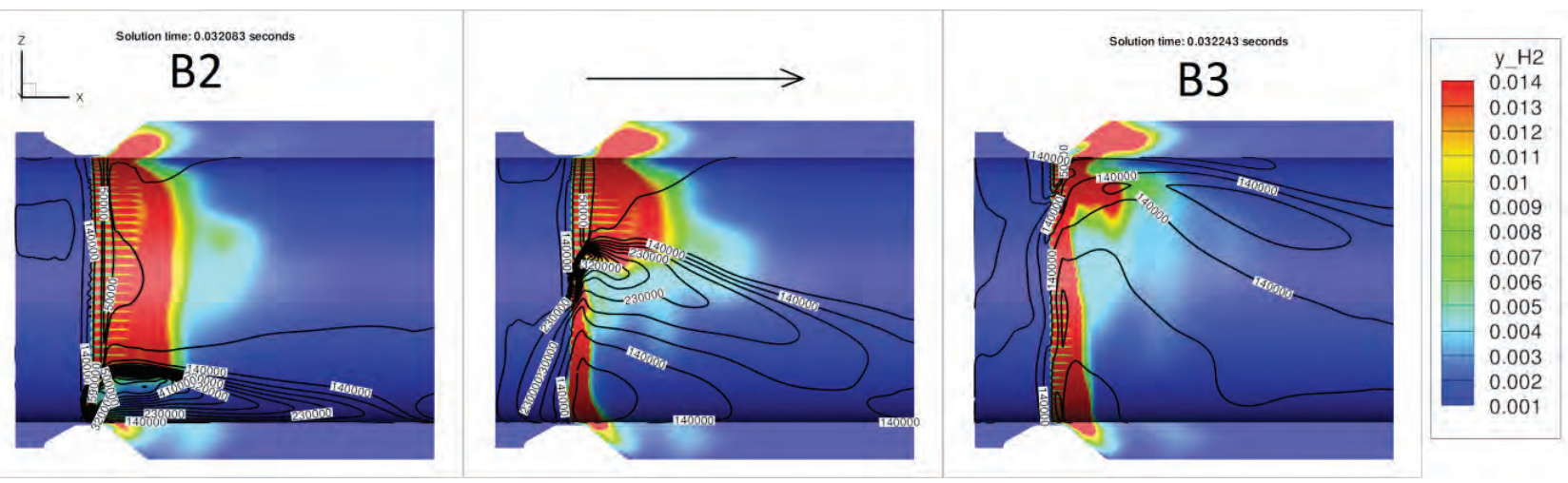

Fig. 5. Contour of hydrogen mass fraction and isolines of static pressure plotted at the cylindrical surface of mean chamber height. B2 and B3 correspond to cases identified in Fig. 4 


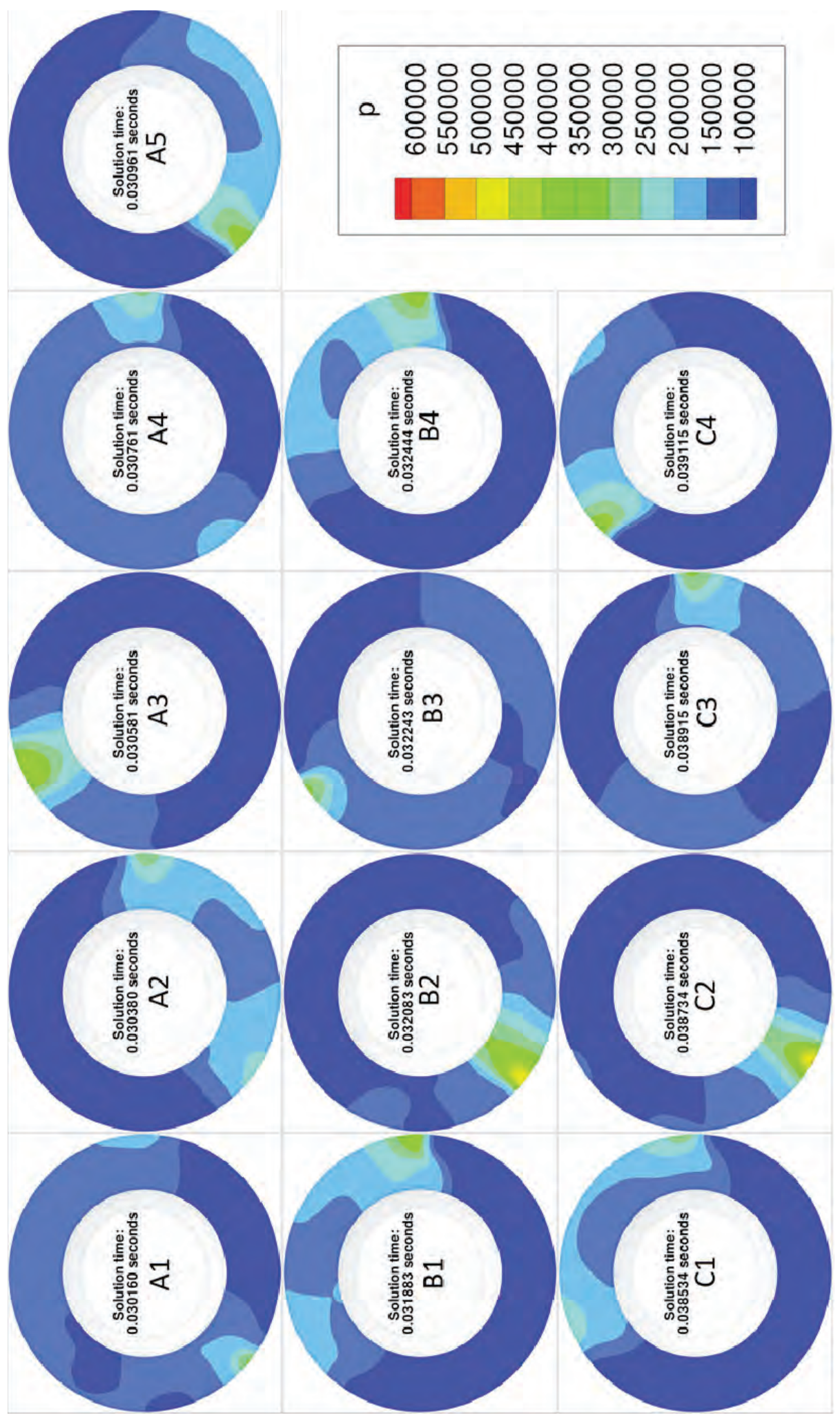

Fig. 6. Contour plot of static pressure in cross section located as shown in Fig. 3. A, B and C correspond to cases identified in Fig. 4

\section{Conclusion}

The above results showed that the rotating detonation can propagate in the RDE chamber in various modes. It was demonstrated that the transition from one mode to another is smooth and the detonation remains stable. Results of simulations quantitatively agree with experimental findings. 


\section{References}

[1] Brown, P. N., Byrne, G. D., Hindmarsh, A. C., VODE: a variable-coefficient ODE solver, Siam J Sci Stat Comput, Vol. 10, No. 5, pp. 1038-1051, 1989.

[2] Folusiak, M., Swiderski, K., Kobiera, A., Wolanski, P., REFLOPS - a New Parallel CFD Code for Reactive Euler Flow Simulation, Arch. Combust., Vol. 29, No. 3-4, pp. 111-152, 2009.

[3] Folusiak, M., Swiderski, K., Wolanski, P., Kobiera, A., Graphics Processors as a Tool for Rotating Detonation Simulations, 23rd International Colloquium on the Dynamics of Explosions and Reactive Systems, University of California, Irvine 2011.

[4] Mott, D. R., Oran, E. S., CHEMEQ2: A Solver for the Stiff Ordinary Differential Equations of Chemical Kinetics, 2001.

[5] Petersen, E. L., Hanson, R. K., Reduced kinetics mechanisms for ram accelerator combustion, J. Propuls. Power, Vol. 15, No. 4, pp. 591-600, 1999.

[6] Tobita, A., Fujiwara, T., Wolanski, P., Detonation engine and flying object provided therewith, United States Patent 7784267, Japanese Patent 2004-1917932010-2005.

[7] Toro, E. F., Riemann Solvers and Numerical Methods for Fluid Dynamics: A Practical Introduction, 3rd ed., Springer, 2009.

[8] Wolanski, P., Detonative propulsion, Proc. Combust. Inst., Vol. 34, No. 1, pp. 125-158, 2013. 